\title{
Use of a decoy peptide to purify p2 I activated kinase-I in cardiac muscle and identification of ceramide-related activation
}

\author{
Yunbo Ke \\ $\mathrm{R}$ John Solaro \\ Department of Physiology \\ and Biophysics, Center \\ for Cardiovascular Research, \\ University of Illinois at Chicago, \\ Chicago, IL, USA
}

Correspondence: Yunbo Ke Department of Physiology and Biophysics, Center for Cardiovascular Research, University of Illinois at Chicago, Chicago, IL 606I2, USA

Tel +I 3129968906

Fax + I 3/2996 I4I 4

Email yke@uic.edu

\begin{abstract}
The $\mathrm{p}^{21}$ activated kinase-1 (Pak1) is a serine-threonine protein kinase directly activated by $\mathrm{Cdc} 42$ and Rac1. In cardiac myocytes, Pak1 activation leads to dephosphorylation of cTnI and C-protein through upregulation of phosphatase-2A (PP2A). Pak1 activity is directly correlated with its autophosphorylation, which occurs upon binding to the small GTPases and to some small organic molecules as well. In this report, we describe a novel method for rapid purification of endogenous Pak1 from bovine ventricle muscle. The method is simple and easy to carry out. The purified Pak1 demonstrated autophosphorylation in vitro that was enhanced by D-erythro-sphingosine-1, $\mathrm{N}$-acetyl-D-erythro-sphingosine $\left(\mathrm{C}_{2}\right.$-ceramide $)$, and $\mathrm{N}$-hexanoylD-erythro-sphingosine ( $\mathrm{C}_{6}$-ceramide). Dihydro-L-threo-sphingosine (saphingol) also had some effect on Pak1 autophosphorylation. The method we developed provides a useful tool to study Pak1 activity and regulation in the heart. Moreover, our results indicate a potential role of the sphingolipids as unique signaling molecules inducing a direct activation of Pak1 that may modulate different cardiac functions.
\end{abstract}

Keywords: pak1, heart muscle, purification, $\mathrm{C}_{2}$ ceramide, $\mathrm{C}_{6}$ ceramide

Despite strong evidence indicating an important role of $\mathrm{p}^{21}$ activated kinase (Pak) in various tissues, detailed understanding of its signaling mechanisms remains poorly understood (Zhao and Manser 2005). An important step in identifying these mechanisms is the development of methods for purification of the enzyme. We describe here a novel approach that we developed for isolation of Pak1 from ventricular myocardium, which will also be useful in other tissues.

Pak1 belongs to a family of serine/threonine protein kinases directly activated by small GTPases, Cdc42 and Rac1. In the heart, Pak1 is abundant and localizes to cell and nuclear membranes, intercalated discs and to Z-discs in ventricular myocytes. The active form of Pak1 in cardiomyocytes increases $\mathrm{Ca}^{2+}$ sensitivity of myofilament force development through activation of PP2A (Ke et al 2004) and in SA nodal cells, Pak1 inhibits isoproterenol stimulated activation of L-type $\mathrm{Ca}^{2+}$ channel and delayed rectifier potassium channels (Ke et al 2007a). Other studies have shown that in endothelial cells, Pak1 activation induces dephosphorylation of myosin regulatory light chain and inhibition of thrombin-induced barrier dysfunction (Ke et al 2007b) and in HeLa cells, expression of constitutively active Pak1 induces loss of stress fibers and dissolution of focal adhesion complexes (Manser et al 1997). These studies suggest a role of Pak1 in cytoskeletal function and reorganization. In transgenic mice expressing an active Rac1 in the heart, hypertrophy developed followed by dilated cardiomyopathy with altered intracellular partitioning of Pak1 in the ventricle myocytes (Sussman et al 2000). 
A prominent post-translational modification of Pak1 is autophosphorylation, which is correlated with its activity (Manser et al 1997; Zhao et al 1998). Pak1 is autophosphorylated at seven serine/threonine sites most of which occur at the N-terminal half of the kinase. Substitution of threonine 423, the last autophosphorylation site, with glutamic acid renders the kinase constitutively active (Manser et al 1997). Although there is abundant expression of Pak1 in cardiomyocytes, smooth muscle and endothelial cells, the function of Pak1 in the cardiovascular system remains poorly understood (Sheehan et al 2007). Moreover, potential modifications in autophosphorylation of native Pak1 in failing heart and in other pathological conditions have not been defined.

Studies in skeletal muscle have shown that Pak1 activity was responsive to insulin treatment (Tsakiridis et al 1996) suggesting that Paks are also phosphorylated by tyrosine kinase (Bagheri-Yarmand et al 2001; He et al 2004; Yang et al 2004). Tyrosine phosphorylation of Pak1 may also play an important role in regulation of cardiac function. However, little is known about any tyrosine kinase signaling mechanism that may play a role in Pak activity.

In order to further study Pak1 function in the heart, we developed a novel affinity chromatography method to purify endogenous Pak1 from heart muscle homogenate. A synthetic peptide derived from Pak1 protein with an HA tag that binds to HA matrix specifically retains Pak1 in the matrix and thus enriches Pak1 from tissue homogenate. A clear, single Pak1 protein band was detected from the eluant. The purified Pak1 demonstrated autophosphorylation that was stimulated by sphingosine and sphingosine derivatives.

\section{Methods}

\section{Preparation of muscle sample}

Frozen bovine ventricle muscle ( $200 \mathrm{~g}$ ) was cut into small pieces and homogenized in a blender containing 1 liter homogenization buffer $(50 \mathrm{mM}$ Tris base, $5 \mathrm{mM}$ EDTA, $2 \mathrm{mM}$ EGTA, $1 \mathrm{mM}$ DTT, $0.5 \mathrm{mM}$ benzamidine, $0.1 \mathrm{mM}$ PMSF, pH 7.2). The homogenized muscle sample was centrifuged at $4500 \mathrm{~g}$ for 30 minutes. The supernatant fraction was saved and precipitated with ammonium sulfate $\left(\mathrm{NH}_{4}\right)_{2} \mathrm{SO}_{4}$ at concentration $25 \%$ and $50 \%(\mathrm{w} / \mathrm{v})$. Precipitates formed by $\left(\mathrm{NH}_{4}\right)_{2} \mathrm{SO}_{4}$ addition between concentration $25 \%(\mathrm{w} / \mathrm{v})$ and $50 \%(\mathrm{w} / \mathrm{v})$ were saved and dialyzed against the homogenization buffer. After dialysis, the sample was filtered and lyophilized. For purification of Pak1 from smaller amounts of sample, the tissues or cells were homogenized in mammalian extraction buffer (50 mM Tris, $\mathrm{pH} 7.5 ; 150 \mathrm{mM} \mathrm{NaCl}$; 0.1\% Nonide P40; Complete Protease inhibitor cocktail tablets [Roche Diagnostics, cat \# 11873580 001]). The ratio of tissue:buffer was about 1:5 $(\mathrm{v} / \mathrm{v})$. The homogenate was centrifuged in a bench-top centrifuge at $4{ }^{\circ} \mathrm{C}$. The supernatant fraction was saved for direct application onto the affinity column.

\section{Peptide synthesis and the matrix}

The peptides were synthesized in the University of Illinois at Chicago protein core facility. The decoy peptide (YNSKKTSNSQKYMSFTDKSAYPYDVPDYA) contained the Pak1 sequence from amino acids 131 to 150 linked to a 9 amino acid of influenza hemagglutinin (HA) epitope. The peptide derived from the N-terminal region of Pak1 with the sequence MSNNGLDIQDKPPAPPMRNTSTYPYDVPDYA was also used in some purification. The Anti-HA Affinity Matrix (Cat. No. 1815 016) was purchased from Roche Applied Science (Indianapolis, IN, USA). The affinity matrix contains antibody specifically bind to HA sequence in protein or peptide.

\section{Affinity chromatography}

The column was first washed with $20 \mathrm{ml}$ equilibration buffer (20 mM Tris, pH 7.5; 0.1 M NaCl; 0.1 mM EDTA). Decoy peptide $(3 \mathrm{mg}$ ) was applied to the column to saturate the matrix with the peptide. The muscle sample (the lyophilized extract) was dissolved in buffer (20 mM Tris, $\mathrm{pH} 7.5 ; 0.1 \mathrm{M}$ $\mathrm{NaCl} ; 0.1 \mathrm{mM}$ EDTA) containing protease inhibitor cocktail tablets. The sample was loaded onto the affinity column and then washed with $20 \mathrm{ml}$ washing buffer $(20 \mathrm{mM}$ Tris, $\mathrm{pH} 7.5 ; 0.1 \mathrm{M} \mathrm{NaCl}$; $0.1 \mathrm{mM}$ EDTA; $0.05 \%$ Tween-20). The Pak1 bound to the gel was eluted with equilibration buffer containing HA peptide (YPYDVPDYA) at $1 \mathrm{mg} / \mathrm{ml}$. HA peptide was removed from the sample by dialysis in buffer containing $20 \mathrm{mM}$ HEPES, $5 \mathrm{mM} \mathrm{NaCl}, \mathrm{pH}$ 7.2. The bound Pak1 could also be eluted with $5 \mathrm{ml}$ glycine buffer, $\mathrm{pH} 2.5$ (50 mM glycine-HCl; $0.1 \%$ Triton X-100; $0.15 \mathrm{M} \mathrm{NaCl}$ ).

\section{Kinase assay}

Pak1 kinase activity was assayed in the following reaction buffer: $50 \mathrm{mM}$ HEPES, pH 7.3, $10 \mathrm{mM} \mathrm{MgCl}, 1 \mathrm{mM}$ DTT, $0.05 \%$ Triton $\mathrm{X}-100$ ) with $10 \mu \mathrm{Ci}$ of $\left[\gamma^{2} \mathrm{P}\right]$ ATP (Perkin Elma. $\left[\gamma_{-}{ }^{32} \mathrm{P}\right]$-Adenosine $5{ }^{`}$-triphosphate, Specific Activity: 3000Ci (111TBq)/mmole, $50 \mathrm{mM}$ Tricine ( $\mathrm{pH} 7.6$ ), Concentration: $5 \mathrm{mCi} / \mathrm{mL}$, Catal NEG502H250UC) at $37{ }^{\circ} \mathrm{C}$ for $2 \mathrm{~h}$. The kinase reaction was stopped by adding SDS sample buffer followed by boiling for $5 \mathrm{~min}$. Agonists added to the buffer were as described in the Results section and the figure legends. Agonists from Calbiochem included the 
following: $\mathrm{C}_{2}$ ceramide ( $\mathrm{N}$-acetyl-D-erythro-sphingosine), $\mathrm{C}_{6}$ ceramide (N-Hexanoyl-D-erythro-sphingosine) and safingol (Dihydro-L-threo-sphingosine). D-sphingosine (S 6879), and sphingosine 1-phosphate (S 9666) were purchased from Sigma (St. Louis, MO, USA). Sphingosines were dissolved in dimethyl sulfoxide. The total reaction volume was $20 \mu \mathrm{l}$. Dimethyl sulfoxide $(2 \mu \mathrm{l})$ was included in each reaction including the control.

\section{Results \\ Cellular localization and purification of Pakl}

Figure 1 show results of immuno-histochemical studies in rat ventricular myocytes demonstrating high and localized expression of Pak1. The bleb in the image of Figure 1A (upper panel) indicates sarcolemmal localization. Pak 1 also localized to the nuclear membrane, Z-discs and intercalated discs (Figure 1A). To isolate the cardiac Pak1, we used bovine ventricle muscle as the source as described in the Methods. About $800 \mathrm{mg}$ of muscle protein extract was obtained from $200 \mathrm{~g}$ of the frozen ventricle muscle. Therefore, the yield of muscle extract from the frozen tissue is about $0.4 \%$. Figure $1 \mathrm{~B}$ illustrates the approach we used for affinity purification of the Pak1 as described in the Methods. The peptide linked affinity column was loaded with $5 \mathrm{mg}$ of the protein extracted from the muscle sample. Data in Figure 2A demonstrate that Pak1 is the major component in the eluted fraction as indicated by the resolution of the samples by SDS-PAGE with Coomassie brilliant blue staining (Figure 3A, lane 3). Ammonia sulfate fractionation decreased amount of some proteins with the molecular weight larger and smaller than Pak1 (Figure 3A). Western blotting analysis (Figure 2B) using an antibody (sc-881) identified the bands shown in Figure 2A as Pak1. The affinity purification procedure yielded about $200 \mu \mathrm{g}$ of total protein from the $5 \mathrm{mg}$ muscle protein extract prepared
A

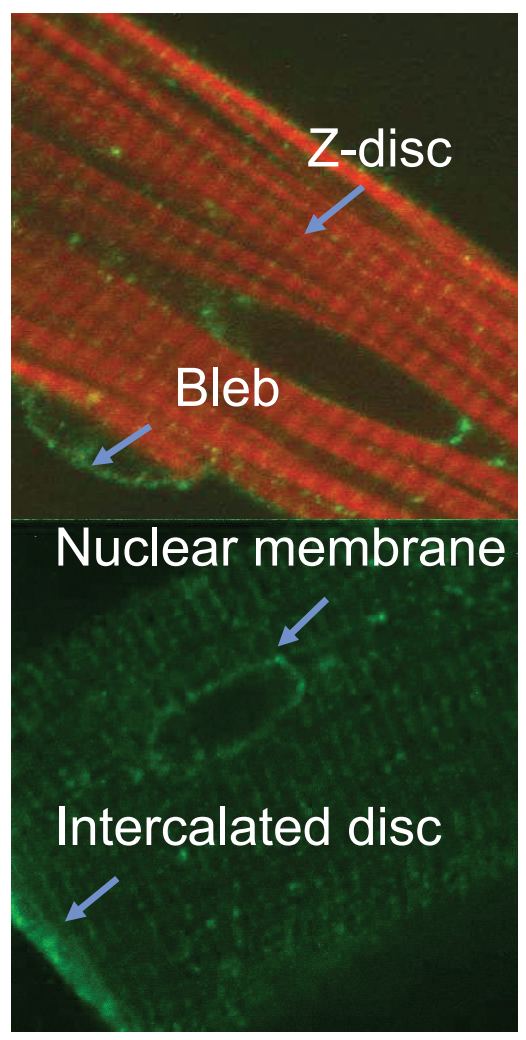

B

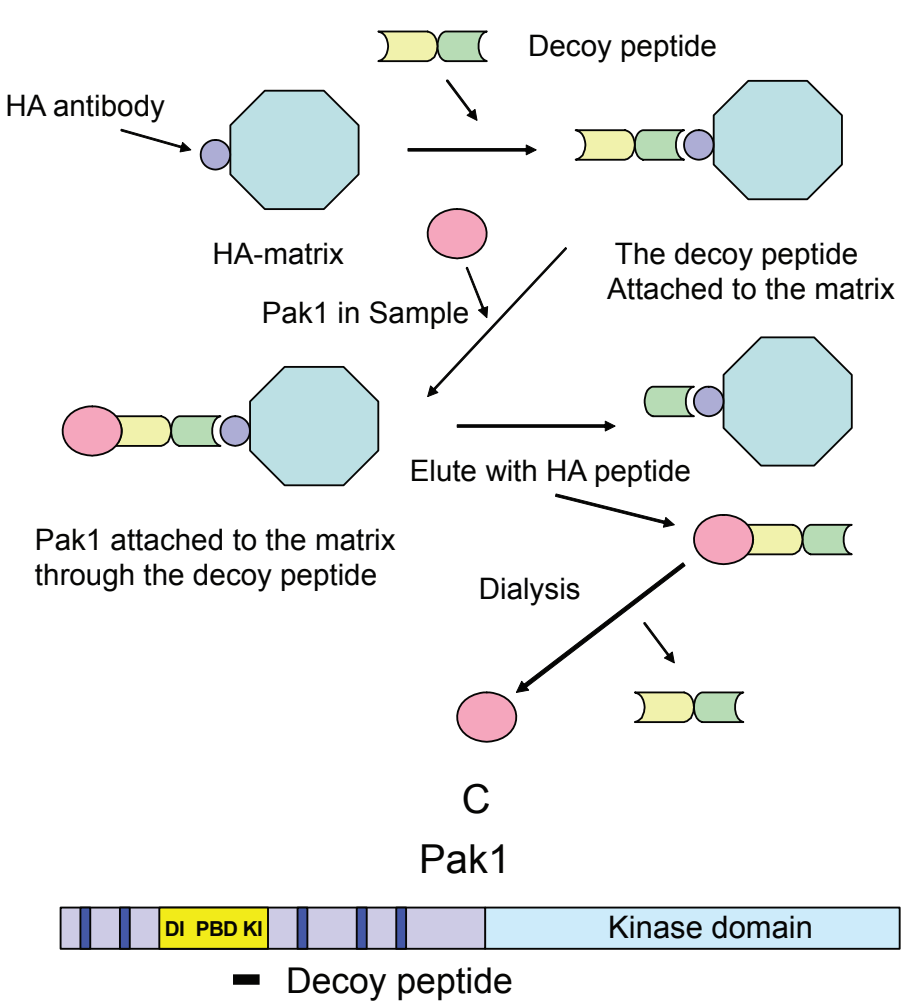

Figure I A. Pak I is abundant and has defined intracellular localizations in adult rat ventricle myocytes. Localization and expression of Pak I in rat ventricle myocytes were detected by immuno-fluorescence and confocal microscopy. Pakl was stained with fluorescein Isothiocyanate (FITC) (green). Actin was stained with phalloidin (red). Pakl is localized to the Z-discs (indicated by the yellow stripes showing overlap of the red and green stains), intercalated discs, nuclear and cell membranes and at the sarcolemmal membrane as emphasized by a bleb in the bottom panel. The myocytes were isolated from rat ventricle as described previously (Ke et al 2004). B. Schematic representation of purification of PakI from cardiac muscle. The purification procedure can be divided into three steps. Step I. The decoy peptide derived from PakI autoinhibitory region linked to influenza hemagglutinin (HA) epitope was bound to HA matrix. Step 2. Pakl was bound to the peptide. The matrix was washed with washing buffer. Step 3. The peptide was eluted out from the matrix by glycine at $\mathrm{pH} 2.5$ or by HA peptide. See text for details. C. The decoy peptide is derived from the autoinhibitory region of Pakl. The autoinhibitory domain of Pakl is downstream of $\mathrm{P}^{21}$ binding domain (PBD) and by dimerization domain (DI). The vertical bars represent the proline rich sequences in Pakl. 
A

\section{$\begin{array}{llll}1 & 2 & 3 & 4\end{array}$}

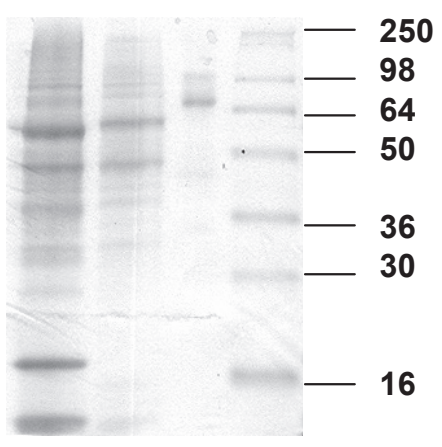

706.4240 (LSAIFR)

웅

C
B

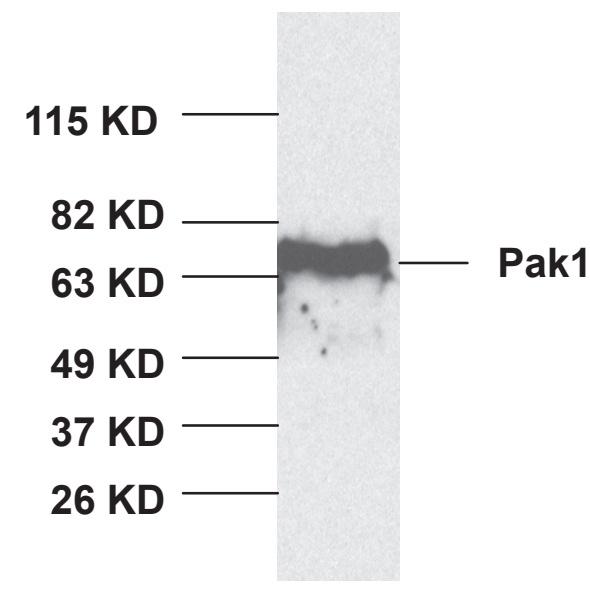

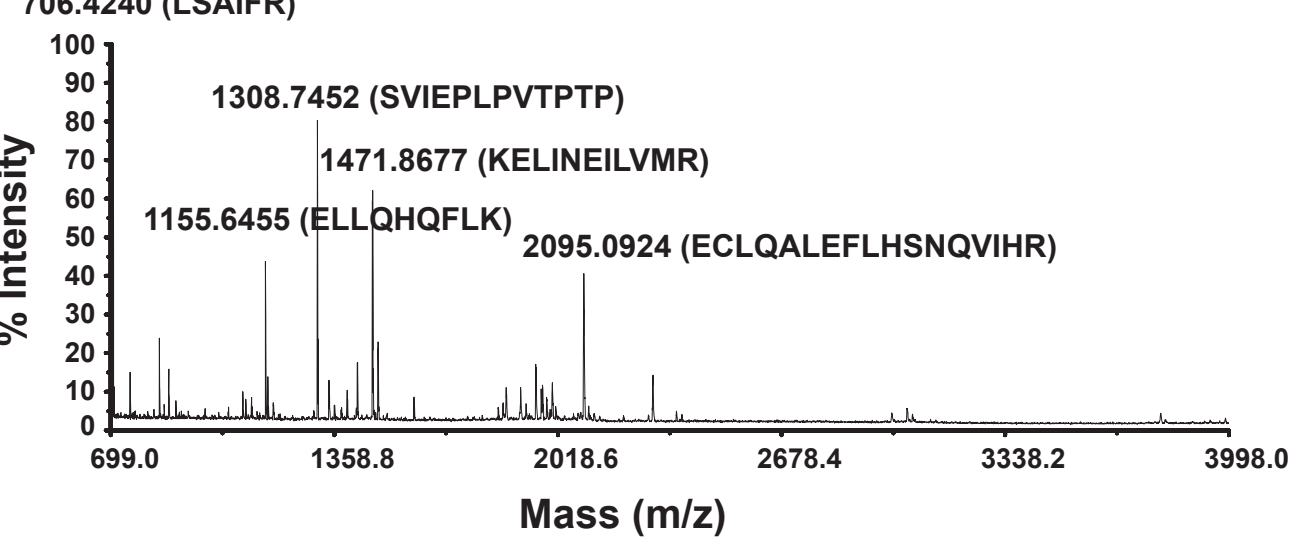

Figure 2 Purified native PakI From cardiac muscle. A. Purification of endogenous PakI from cardiac muscle. I. Cardiac muscle extracts resolved on a SDS page. 2. The cardiac muscle extract after fractionation by $25 \%$ and $50 \%\left(\mathrm{NH}_{4}\right)_{2} \mathrm{SO}_{4}$ precipitation. 3. Pakl purified from the fractionated muscle sample. 4 . The molecular weight markers. B. Detection of purified Pakl by Western blotting analysis. Pakl was detected as a single band with an antibody from Santa Cruz (sc-88I). C. Purified PakI detected by mass spectrometry. Peaks match the Pakl peptides produced by theoretical trypsin digestion. The positions of amino acids of each peptide from Pakl were denoted in quotation: LSAIFR (490-495), ELLQHQFLK (5I4-522), SVIEPLPVTPTR (204-2I5), KELIINEILVMR (309-320), ECLQALEFLHSNQVIHR (372-388).

\section{A}

\section{Sp-1-P $00 \quad 0 \quad 2550100200 \mu M$ Pak1 $\begin{array}{lll}0 & 4 & 4\end{array}$}

D-Sphingosine Pak1

B

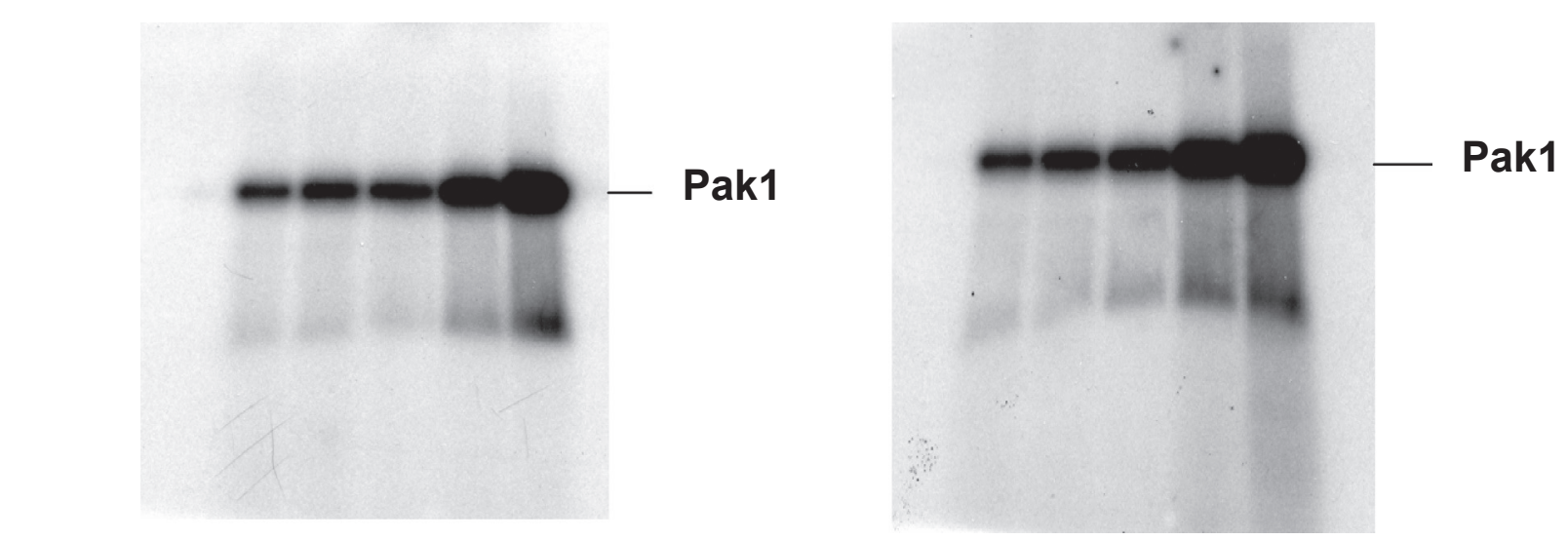
$\begin{array}{llllll}0 & 25 & 50 & 100 & 200 & \mu M \\ 4 & 4 & 4 & 4 & 4 & \mu g\end{array}$

Figure 3 A.Autophosphorylation of Pakl is stimulated by sphingosine-phosphate in vitro. The in vitro reaction was carried out at $37^{\circ} \mathrm{C}$ for two hours. The same amount of Pakl protein $(4 \mu \mathrm{g})$ was included in each reaction. Sphingosine phosphate was added to each reaction at concentrations $0,25,50,100$, and $200 \mu \mathrm{M}$. B.Autophosphorylation of Pakl is stimulated by D-erythro-sphingosine in vitro. The in vitro reaction was carried out at $37^{\circ} \mathrm{C}$ for two hours. $4 \mu \mathrm{g}$ of Pakl protein was included in each reaction. Increasing amounts of D-erythro-sphingosine were added at $0,25,50,100$, and $200 \mu \mathrm{M}$. The smaller band below $68 \mathrm{kD}$ Pakl signal is about $48 \mathrm{kD}$ and is a Pakl degradation product. 
from the frozen ventricle with a yield of $4 \%$. Therefore, the yield of final affinity purification product from the frozen ventricle is about $0.016 \%(\mathrm{w} / \mathrm{w})$. Pak1 bound to the matrix was eluted with $3 \mathrm{mg}$ of HA peptide that displace the decoy peptide from the matrix. The HA peptide in the eluant was separated from Pak1 protein by use of dialysis tubing permeable to proteins smaller than $12-14 \mathrm{kD}$ (Figure 1B). The bound Pak1 can also be eluted with Glycine (0.1 M, pH2.0). However, elution with glycine sometimes produces an extra protein bands with molecular weight of $16 \mathrm{kD}$. Mass spectrometry analysis indicated that the major MS peaks from the digested protein purified matches with Pak1 peptides after trypsin digestion (Figure 2C).

\section{In vitro autophosphorylation}

Autophosphorylation of Pak1, which correlates with its activity and that we assessed with an in vitro assay, provided a measure of Pak-1 enzyme function. Initial studies (data not shown) indicated that autophosphorylation of the wt Pak1 was much weaker than the constitutively active Pak1 as shown by others (Manser et al 1997). In all the in vitro assays, we used $4 \mu \mathrm{g}$ of Pak1 protein. The smaller band $(\sim 48 \mathrm{kD})$ that could be Pak1 degradation products was also phosphorylated. The purified Pak1 also demonstrated kinase activity towards the decoy peptide (YNSKKTSNSQKYMSFTDKSA). The decoy peptide contains Pak1 autophosphorylation sites.
The two serine residues that are autophosphorylated are situated at the end of the autoinhibitory sequence. The decoy peptide slightly inhibited autophosphorylation of Pak1. However, it was also phosphorylated and there was a slight increase of the total kinase activity in the presence of the decoy peptide (data not shown).

In view of data reporting a sphingosine-1 induced increase in Pak1 and Pak2 activity in vitro (Bokoch et al 1998; Roig et al 2001), we also tested the effect of sphingosine and sphingosine-1 phosphate on Pak1 autophosphorylation (Figure 3A). Incorporation of 32P from gamma 32P into Pak1 was directly detected by autoradiography after resolving the in vitro reaction products on SDS PAGE. Interestingly, sphingosine-1 phosphate had no significant effect on autophosphorylation of Pak1 at 25 and $50 \mu \mathrm{M}$. When the sphingosine phosphate concentration increased to 100 and $200 \mu \mathrm{M}$, there was an increase in Pak1 autophosphorylation. In purified Pak1 sample, a 48 kD Pak1 degradation products can sometimes be detected (Figure 1A, lane 3). When Pak1 activity increased, phosphorylation of the degradation products increased accordingly (Figure 3). Our results indicated that autophosphorylation of Pak1 purified from cardiac muscle was highly responsive to $\mathrm{C}_{2}$ and $\mathrm{C}_{6}$ ceramide (Figure $4 \mathrm{~A}$ and $\mathrm{B}$ ). Autophosphorylation of the purified Pak1 increased in the presence of 50 and $100 \mu \mathrm{M}$ of D-sphingosine-1 (Figure 3B), but saphingol (dihydro-L-threo-sphingosine) only slightly

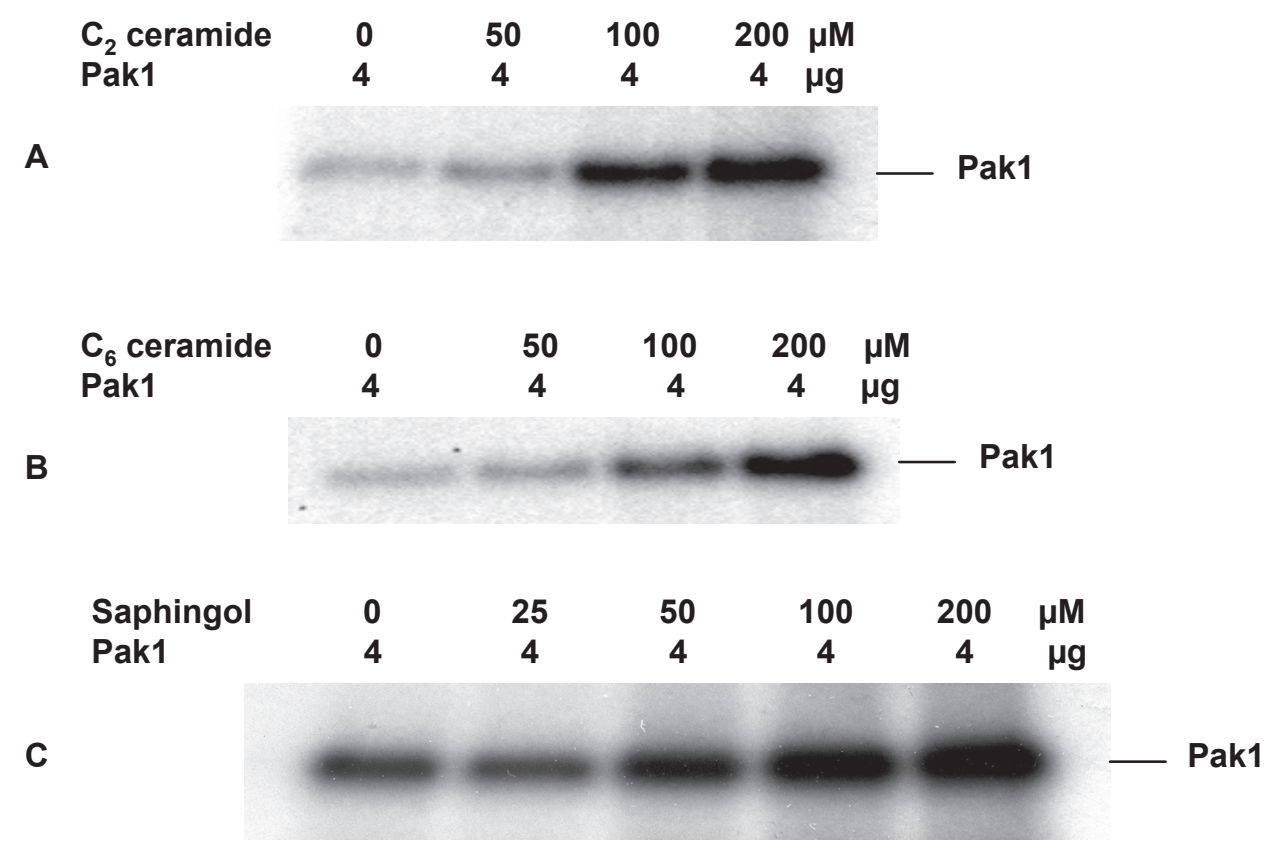

Figure $4 \mathrm{~A}$ and $\mathbf{B}$. Autophosphorylation of Pakl is stimulated by $C_{2}$ (left) and $C_{6}$ ceramide (right) in vitro. $C_{2}$ and $C_{6}$ ceramide was added to the reaction at different concentrations at 0,50, 100, and $200 \mu \mathrm{M}$. Pakl protein $(4 \mu \mathrm{g})$ was included in each reaction. In $\mathbf{C}$, autophosphorylation of Pakl is regulated to a lesser extent by saphingol (dihydro-sphingosine). Saphingol was added to each reaction at concentrations of 0,25, 50, 100, and $200 \mu \mathrm{M}$. 
regulated Pak1 autophosphorylation (Figure 4C). By gel filtration, the purified Pak1 demonstrated a single major peak. The same experiments were repeated three times each.

\section{Discussion}

Our data demonstrating a simple method for purifying native Pak1 from cardiac muscle provide significant new information important in advancing understanding of the Pak-1 signaling in the heart. Moreover, to our knowledge, our data also provide the first evidence demonstrating regulation of the activity of Pak1 by $\mathrm{C}_{2}$ and $\mathrm{C}_{6}$ ceramide. Our understanding of Pak1 function in myocardial cells is still in its infancy, and we think the method reported here is an important step in defining these functions and their mechanisms. Signaling molecules such as Pak1 are often extensively modified by post-translational mechanisms, and in many cases these modifications are associated with pathophysiological conditions. Our method is suitable for study of post-translational modifications of Pak1 in response to different extracellular signals and in pathological conditions such as heart failure, arrhythmias, and ischemia. The Pak1 kinase purified by this method can also be used for in vitro kinase assay to identify novel activators or inhibitors. Moreover, the method can be adapted for use in purification of Pak isoforms from mammalian tissues other than heart.

The purified Pak1 retained low catalytic activity as demonstrated by autophosphorylation in vitro. The constitutively active Pak1 (T423E) has a much higher level of autophosphorylation than the endogenous Pak1 under the same conditions. We found that autophosphorylation of the purified Pak1 is enhanced by D-sphingosine-1, N-acetyle-sphingosine $\left(\mathrm{C}_{2}\right.$ ceramide $)$ and by hexanoyl-sphingosine $\left(\mathrm{C}_{6}\right.$ ceramide $)$. This is consistent with an earlier observation that the activities of Pak1 and Pak2 are activated by sphingosine (Bokoch et al 1998; Roig et al 2001). Our finding that active Pak1 induces dephosphorylation of cTnI and C-protein through activation of PP2A (Ke et al 2004) provides a plausible mechanism for induction of protein dephosphorylation by sphingosine. For example, $\mathrm{C}_{2}$-ceramide is a known activator of PP2A (Dobrowsky et al 1993) and may be involved in Bcl2 dephosphorylation (Ruvolo et al 1999). In different types of cells, L-type $\mathrm{Ca}^{2+}$ channel activity was demonstrated to be depressed by $\mathrm{C}_{2}$ ceramide by an unknown mechanism (Chik et al 1999, 2004). $\mathrm{C}_{2}$ ceramide also inhibited proliferation of T-cells stimulated by growth signals (O'Byrne and Sansom 2000). Moreover, $\mathrm{C}_{2}$ ceramide has preconditioning effects in heart, which has been attributed to generation of reactive oxygen species (Furuya et al 2001; Lecour et al 2006).
Our data indicate that other mechanisms for $\mathrm{C}_{2}$ ceramide involving activation of Pak1 and PP2A need to be considered. To our knowledge, this is the first time demonstration that Pak1 is directly stimulated by $\mathrm{C}_{2}$ and $\mathrm{C}_{6}$ ceramide. The mechanism whereby Pak1 is activated by sphingosine analogs is still not clear. Our studies indicate that $\mathrm{C}_{2}$ and $\mathrm{C}_{6}$ ceramide had stronger effect than sphingosine-1 activating Pak1. Saphingosine only had a small stimulatory effect on Pak1 autophosphorylation. Zenke and colleagues (1999) suggest that sphingosine activates Pak through the P21 binding domain (PBD). However, in another study, Cdc42 and sphingosine-1 appears to have a cooperative effect activating the kinase (Chong et al 2001). We plan to employ a molecular biology approach to map the amino acid sequence on Pak1 that interacts with sphingosines.

The peptide used to decoy native Pak1 from cardiac muscle is derived from the autoinhibitory region of Pak1 (Manser et al 1997) (Figure 1C). The autoinhibitory region is both downstream of and partially overlaps with the $\mathrm{P}^{21}$ binding domain (PBD) (Zhao et al 1998). A peptide derived from the N-terminal region (the first 21 amino acids of Pak1) that binds to Nck in vitro (Bokoch et al 1998; Zhao et al 2000) also efficiently retained Pak1 in the column (data not shown). The peptide interacts with the catalytic center of the kinase and may regulate the conformation of the catalytic center as demonstrated by NMR studies (Pirruccello et al 2006). Expression of the peptide plus the $\mathrm{P}^{21}$ binding region produced many cellular changes including inhibition of cell cycle progression (Thullberg et al 2007). The autoinhibitory region was also deleted in GST-Pak1 which was used for in vitro kinase studies (Polverino et al 1995).

Pak1 is autophosphorylated at multiple serine/threonine sites. There are at least six autophosphorylation sites at the N-terminal half of Pak1 (Manser et al 1997). Autophosphorylation at the N-terminus of Pak1 may change its intracellular localization or/and activity (Zhao and Manser 2005). It is unclear at which site autophosphorylation occurs first in the activation of Pak1. Furthermore, uncertainty still remains as to whether autophosphorylation is through interor intramolecular kinase reactions. Since Pak1 dimer has an antiparallel conformation, autophosphorylation sites at the N-terminal half of one Pak1 are therefore placed in proximity to the catalytic domain of the other Pak1 (Lei et al 2000). This suggests that intermolecular autophosphorylation may occur at the N-terminal autophosphorylation sites.

Pak1 contains a few proline-rich sequences at its $\mathrm{N}$-terminus. The proline-rich sequences interact with proteins, such as Nck and Pix. A prominent feature of these 
proline-rich sequences in Pak1 is that they are followed by autophosphorylation sites. Peptide decoy was first designed to identify proteins that interact with the proline rich sequences in the heart. It turned out Pak1 is the only major protein that binds to the decoy peptides, including the peptide containing 22 amino acids (MSNNGLDIQDKPPAPPMRNTST) at the N-terminal region of the kinase which contains an autophosphorylation site at Serine 21. Substitution of the serine residue for aspartic acid abolished Nck binding to the peptide in vitro (Zhao et al 2000). In vivo, phosphorylation at the autophosphorylation sites could be a mechanism to release the "autosubstrate" from the catalytic center of the kinase.

Interaction between lipids and a protein kinase may have profound effect on cellular functions (Argraves et al 2002). The purified Pak1 is stimulated by more than one sphingosine species. It is likely that some other sphingosine related lipids may also regulate Pak1 activity. Modification of the side chain and the polar groups of sphingosines may produces agonists or antagonists that have even more potent effect on Pak1 autophosphorylation.

\section{Acknowledgments}

These experiments were supported by PO1 HL 62426 (RJS) and by R01 HL64035 (RJS). We thank Bao-Shiang Lee in UIC Research Resources Center for technical assistance, Nicole Glaser (NIA) for critical reading of the manuscript and Joyce W Ke for preparation of the Pak1 samples. The authors report no conflicts of interest in this work.

\section{References}

Argraves KM, Obeid LM, Hannun YA. 2002. Sphingolipids in vascular biology. Adv Exp Med Biol, 507:439-44.

Bagheri-Yarmand R, Mandal M, Taludker AH, et al. 2001. Etk/Bmx tyrosine kinase activates Pak1 and regulates tumorigenicity of breast cancer cells. J Biol Chem, 276:29403-9.

Bokoch GM, Reilly AM, Daniels RH, et al. 1998. A GTPase-independent mechanism of $\mathrm{p} 21$-activated kinase activation. Regulation by sphingosine and other biologically active lipids. J Biol Chem, 273:8137-44.

Chik CL, Li B, Karpinski E, et al. 2004. Ceramide inhibits L-type calcium channel currents in GH3 cells. Mol Cell Endocrinol, 218:175-83.

Chik CL, Li B, Negishi T, et al. 1999. Ceramide inhibits L-type calcium channel currents in rat pinealocytes. Endocrinology, 140:5682-90.

Chong C, Tan L, Lim L, et al. 2001. The mechanism of PAK activation. Autophosphorylation events in both regulatory and kinase domains control activity. $J$ Biol Chem, 276:17347-53.

Dobrowsky RT, Kamibayashi C, Mumby MC, et al. 1993. Ceramide activates heterotrimeric protein phosphatase 2A. J Biol Chem, 268:15523-30.

Furuya K, Ginis I, Takeda H, et al. 2001. Cell permeable exogenous ceramide reduces infarct size in spontaneously hypertensive rats supporting in vitro studies that have implicated ceramide in induction of tolerance to ischemia. J Cereb Blood Flow Metab, 21:226-32.
He H, Hirokawa Y, Gazit A, et al. 2004. The Tyr-kinase inhibitor AG879, that blocks the ETK-PAK1 interaction, suppresses the RAS-induced PAK1 activation and malignant transformation. Cancer Biol Ther, 3:96-101.

Ke Y, Lei M, Collins TP, et al. 2007. Regulation of L-type calcium channel and delayed rectifier potassium channel activity by 21 -activated kinase-1 in guinea pig sinoatrial node pacemaker cells. Circ Res, 100:1317-27.

Ke Y, Lum H, Solaro RJ. 2007. Inhibition of endothelial barrier dysfunction by P21-activated kinase-1. Can J Physiol Pharmacol, 85:281-8.

Ke Y, Wang L, Pyle WG, et al. 2004. Intracellular localization and functional effects of P21-activated kinase-1 (Pak1) in cardiac myocytes. Circ Res, 94:194-200.

Lecour S, Owira P, Opie LH. 2006. Ceramide-induced preconditioning involves reactive oxygen species. Life Sci, 78:1702-6.

Lei M, Lu W, Meng W, et al. 2000. Structure of PAK1 in an autoinhibited conformation reveals a multistage activation switch. Cell, 102:387-97.

Manser E, Huang HY, Loo TH, et al. 1997. Expression of constitutively active alpha-PAK reveals effects of the kinase on actin and focal complexes. Mol Cell Biol, 17:1129-43.

O'Byrne D, Sansom D. 2000. Lack of costimulation by both sphingomyelinase and $\mathrm{C} 2$ ceramide in resting human $\mathrm{T}$ cells. Immunology, 100:225-30.

Pirruccello M, Sondermann H, Pelton JG, et al. 2006. A dimeric kinase assembly underlying autophosphorylation in the p21 activated kinases. J Mol Biol, 361:312-26.

Polverino A, Frost J, Yang P, et al. 1995. Activation of mitogen-activated protein kinase cascades by $\mathrm{p} 21$-activated protein kinases in cell-free extracts of Xenopus oocytes. J Biol Chem, 270:26067-70.

Roig J, Tuazon PT, Traugh JA. 2001. Cdc42-independent activation and translocation of the cytostatic $\mathrm{p} 21$-activated protein kinase gamma-PAK by sphingosine. FEBS Lett, 507:195-9.

Ruvolo PP, Deng X, Ito T, et al. 1999. Ceramide induces Bcl2 dephosphorylation via a mechanism involving mitochondrial PP2A. J Biol Chem, 274:20296-300.

Sheehan KA, Ke Y, Solaro RJ. 2007. p21-Activated kinase-1 and its role in integrated regulation of cardiac contractility. Am J Physiol Regul Integr Comp Physiol, 293:R963-73.

Sussman MA, Welch S, Walker A, et al. 2000. Altered focal adhesion regulation correlates with cardiomyopathy in mice expressing constitutively active rac1. J Clin Invest, 105:875-86.

Thullberg M, Gad A, Beeser A, et al. 2007. The kinase-inhibitory domain of p21-activated kinase 1 (PAK1) inhibits cell cycle progression independent of PAK1 kinase activity. Oncogene, 26:1820-8.

Tsakiridis T, Taha C, Grinstein S, et al. 1996. Insulin activates a p21-activated kinase in muscle cells via phosphatidylinositol 3-kinase. J Biol Chem, 271:19664-7.

Yang Z, Bagheri-Yarmand R, Wang RA, et al. 2004. The epidermal growth factor receptor tyrosine kinase inhibitor ZD1839 (Iressa) suppresses c-Src and Pak1 pathways and invasiveness of human cancer cells. Clin Cancer Res, 10:658-67.

Zenke FT, King CC, Bohl BP, et al. 1999. Identification of a central phosphorylation site in p21-activated kinase regulating autoinhibition and kinase activity. J Biol Chem, 274:32565-73.

Zhao ZS, Manser E. 2005. PAK and other Rho-associated kinases - effectors with surprisingly diverse mechanisms of regulation. Biochem $J$, 386:201-14.

Zhao ZS, Manser E, Chen XQ, et al. 1998. A conserved negative regulatory region in alphaPAK: inhibition of PAK kinases reveals their morphological roles downstream of $\mathrm{Cdc} 42$ and Rac1. Mol Cell Biol, 18:2153-63.

Zhao ZS, Manser E, Lim L. 2000. Interaction between PAK and nck: a template for Nck targets and role of PAK autophosphorylation. Mol Cell Biol, 20:3906-17. 
